\section{Agonist-antagonist opioids: theory and clinical practice}

This presentation will deal with the heterogeneous group of opioid analgesics possessing opioid antagonist properties. The clinically available drugs of this class are pentazocine, butorphanol, nalbuphine, buprenorphine, and meptazinol (in the UK). At this writing, dezocine has completed human trials and awaits FDA approval in the US.

The prototype agonist-antagonist, $\mathrm{N}$-allylnormorphine (nalorphine), was tested in animals and shown to be a morphine antagonist as early as 1930. Nalorphine was thought to be devoid of analgesic effects until the early 1950's when Lasagna and Beecher discovered that it had analgesic potency comparable to morphine.' They noted that it produced typical morphine-like effects such as miosis and respiratory depression, but it also produced unexpected effects like dysphoria and hallucinations.

At nearly the same time Wikler and others showed that nalorphine could precipitate a violent withdrawal syndrome in opioid-dependent individuals. ${ }^{2}$ When it was given to former heroin addicts they experienced no euphoria or drug craving.

The dysphoric effects and hallucinations made nalorphine unacceptable as an analgesic (although it was used for years as an antagonist). It is much more significant that nalorphine was the first potent analgesic which had little or no addictive liability. The pharmaceutical industry was motivated to synthesize and test thousands of nalorphine congeners, and a few of these have proved to be useful analgesics with a very low potential for abuse.

Thus far, the agonist-antagonist analgesics have not attained widespread popularity in anaesthetic practice. I attribute this to three factors:

1 Much of the research done on these agents is not directly applicable to the anaesthetic setting.

2 There is still widespread misunderstanding of agonistantagonist pharmacology. These drugs were initially marketed as less abusable substitutes for morphine and fentanyl. Many anaesthetists were surprised to discover that they differed from the pure agonists in some important ways. For some perioperative uses of morphine or fentanyl the agonist-antagonist analgesics were probably not satisfactory substitutes.

3 The advantage of low-abuse potential is (unfortunately) not generally perceived to be relevant to our specialty.
Carl Rosow MD

The diversion and abuse of fentanyl by anaesthetic personnel was not widely appreciated until recently.

\section{Mechanism of action}

When an opioid binds to its receptor it may produce a variable degree of effect: A full agonist like fentanyl or morphine can produce intense analgesia and respiratory depression, while a competitive antagonist like naloxone binds with high affinity but produces little or no effect. Nalorphine and all of the other agonist-antagonists behave as partial agonists; these drugs tend to have shallow dose-response curves and produce lower maximal effects. Although this means that there is a "ceiling" to the analgesic effects, toxic effects are limited as well. Keats and Telford showed, for example, that increasing doses of nalorphine produced very limited increases in respiratory depression. ${ }^{3}$

Martin and his coworkers have postulated that the opioid alkaloids interact with (at least) three different receptors called mu, kappa, and sigma (Table I). ${ }^{4}$ Each opioid may act as an agonist, a partial agonist or an antagonist at each receptor (Table II). Nalorphine, pentazocine, butorphanol, and nalbuphine are thought to produce their analgesic and sedative effects by interacting with kappa receptors. Each of them has affinity, but no efficacy, at mu receptors and therefore acts as a morphine antagonist. Dysphoria and hallucinations are thought to be mediated by sigma receptors.

Buprenorphine, dezocine, and meptazinol also have agonist and antagonist effects, but they are thought to be partial agonists at mu receptors. Buprenorphine has been shown to have extremely high affinity and relatively low efficacy at mu receptors. When given alone, it produces effects which appear similar to morphine. When given after morphine, it displaces the full agonist and causes a reduction in opioid effect.

As you might imagine, reversal of morphine with a mu or kappa partial agonist is not as straightforward as reversal with naloxone. In the presence of a fixed amount of morphine a small dose of nalorphine may antagonize

Massachusetts General Hospital, Anaesthesia Dept., Fruit Street, Boston, MA 02114, (617) 726-8812. 
TABLE I Opiojd receptor subtypes adapted from Martin (Ref. 4)

\begin{tabular}{llll}
\hline & \multicolumn{2}{l}{ Receptor } & \\
\cline { 2 - 3 } Response & mu & kappa & sigma \\
\hline analgesia & yes & yes & no \\
respiratory depression & yes & yes & no \\
behaviour & euphoria & sedation & dysphoria \\
pupil constriction & yes & yes & no \\
morphine subsitution & yes & no & no \\
\hline
\end{tabular}

Table II Receptor interactions* for various opioids

\begin{tabular}{llll}
\hline & \multicolumn{2}{l}{ Receptor } & \\
\cline { 2 - 4 } Drug & $m u$ & kappa & sigma \\
\hline Fentanyl & $\mathrm{Ag}$ & - & - \\
Morphine & $\mathrm{Ag}$ & $\mathrm{Ag}$ & - \\
& & & \\
Nalorphine & $\mathrm{Antag}$ & $\mathrm{PAg}$ & $\mathrm{Ag}$ \\
Pentazocine & $\mathrm{Antag}$ & $\mathrm{P} \mathrm{Ag}$ & $\mathrm{Ag}$ \\
Butorphanol & $\mathrm{Antag}$ & $\mathrm{PAg}$ & $\mathrm{P} \mathrm{Ag}$ \\
Nalbuphine & $\mathrm{Antag}$ & $\mathrm{PAg}$ & $\mathrm{PAg}$ \\
& & & \\
Buprenorphine & $\mathrm{P} \mathrm{Ag}$ & - & - \\
Dezocine & $\mathrm{P} \mathrm{Ag}$ & - & - \\
Meptazinol & $\mathrm{P} \mathrm{Ag \dagger}$ & - & - \\
\hline
\end{tabular}

*Ag = Agonist; P Ag = Partial Agonist; Antag = Antagonist. tOther mechanisms probably play a role in the analgesic effect.

the opiate effects, while a large dose may actually increase them. The net effect therefore depends upon the ratio of morphine to nalorphine. ${ }^{5}$

These drugs vary widely in antagonist and agonist potency. Neither agonist vs. antagonist potency nor hypothetical receptor interaction has proved to be a good predictor of clinical utility or patient acceptance.

\section{Clinical pharmacology}

\section{Analgesia}

The clinically available agonist-antagonists possess sufficient analgesic activity to be effective in a variety of acute and chronic pain states. They have been shown to be sufficient for treatment of postoperative pain, trauma, burns, labour pain, renal colic, etc. They have been given intramuscularly, orally, sublingually, intranasally, intravenously by bolus or continuous infusion, and in patientcontrolled analgesia systems. Several have been used successfully by the epidural route. The initial reports on epidural butorphanol after Caesarean section seem promising: Abboud reported that it produces excellent pain relief and little or no pruritus. ${ }^{6}$

Agonist-antagonists can be used successfully as part of a balanced anaesthetic technique, but here their partial agonist properties are more evident. Given alone, even extremely large doses of these drugs will usually not produce a state of "anaesthesia" as might be seen with fentanyl or its derivatives. ${ }^{7}$ Compared with fentanyl, the agonist-antagonists produce more limited decreases in requirements for potent volatile anaesthetics. ${ }^{3}$

\section{Sedation and mood effects}

A morphine-like agonist produces pain relief which is usually accompanied by dissociation, drowsiness, and mood elevation. As the dose is raised there is progressively more mental clouding, lethargy, and stupor. Patients given the kappa-type agonist-antagonist pentazocine may experience floating and dissociation, but usually not mood elevation. ${ }^{9}$ They often appear extremely sedated yet remain capable of suprisingly lucid conversation. Sometimes the sedation will appear greater than the self-described relief of pain. With higher doses patients are much more likely to experience "weird" feelings, depersonalization, or hallucinations. The dysphoric effects are much more prominent with pentazocine than with butorphanol or nalbuphine. The subjective effects of the mu partial agonists (buprenorphine and dezocine) are morphine-like throughout the dose range.

These mood effects have important therapeutic implications. Most physicians are very familiar with the pleasant mental detachment produced by morphine and use it as a sign of drug effect. Since euphoria does not usually occur with the agonist-antagonists there is a tendency to ascribe lack of mood elevation to lack of analgesia. Kaiko et al. showed that these two effects are separable in a multi-dose comparison of meptazinol and morphine in postoperative pain. ${ }^{10}$ Using a standard postoperative pain model they showed that both drugs produced dose-related pain relief, but only morphine produced dose-related improvement in mood. It is important to remember that abuse of morphine is largely attributable to its euphoriant effects. While we want these drugs to make our patients feel better, it is not clear whether mood elevation is always necessary or desirable in an analgesic.

The prominent sedative effects of butorphanol or nalbuphine may be advantageous in some clinical situations. Butorphanol has shown promise as a sedative either alone or in combination with a benzodiazepine. "In my own practice, small doses (1-4 mg) of butorphanol, with or without midazolam, are useful adjuncts to regional anaesthetic techniques. The lower end of this dose range is suitable for elderly patients and those scheduled for discharge the same day.

\section{Respiratory depression}

Much has been made of the limited respiratory depression exhibited by these drugs. Respiratory depression after 2 or 
$4 \mathrm{mg}$ of butorphanol is equivalent to that produced by 10 $\mathrm{mg}$ of morphine. ${ }^{12}$ Respiratory depression reaches a maximum after about $30 \mathrm{mg}$ of nalbuphine. ${ }^{13}$ Even larger doses are well tolerated by most patients, but severe respiratory embarrassment can still be produced in sensitive individuals and in those with concomitant pulmonary or central nervous system disease. Respiratory depression may be reversed with naloxone.

A ceiling effect on respiration has also been demonstrated for buprenorphine in animals, and it probably occurs in humans. This is important, since buprenorphine is not reliably antagonized by naloxone. ${ }^{14}$ The other mu partial agonist, dezocine, has substantially higher intrinsic activity than the other agonist-antagonists, ${ }^{15}$ and it produced apnoea in one of our study patients (Rosow, unpublished observations).

\section{Smooth muscle effects}

The kappa type agonist-antagonists have all been shown to produce much less spasm of gastrointestinal smooth muscle than morphine. Pentazocine, nalbuphine, and butorphanol do not cause appreciable elevation of intrabiliary pressure, and they may be particularly useful in patients who experience biliary colic after morphine. ${ }^{16}$

\section{Cardiovascular effects}

Pentazocine and butorphanol have been reported to cause unique cardiovascular effects in animals and in patients with cardiovascular disease. The mechanism(s) for these effects are unknown. They have not been reported to occur with nalbuphine or buprenorphine.

In contrast to morphine, pentazocine may increase heart rate, systemic and pulmonary arterial pressure, and cardiac work. ${ }^{17}$ It is likely that myocardial oxygen consumption increases, so pentazocine may be a poor choice in the setting of myocardial ischaemia or infarction.

Butorphanol has been reported to increase pulmonary artery pressure, while heart rate and systemic pressure usually decrease slightly. ${ }^{18}$ High doses have been given safely to patients undergoing coronary bypass grafting, ${ }^{19}$ although other opioids may be more desirable in this setting.

\section{Antagonist effects}

Nalbuphine and buprenorphine are relatively strong antagonists and have been used clinically for this purpose. ${ }^{20}$ I have not seen convincing evidence that reversal with an agonist-antagonist is safer or more reliable than with naloxone.

Therapeutic doses of pentazocine, nalbuphine and buprenorphine unequivocally precipitate withdrawal in opioid-dependent individuals. Butorphanol seems to be much weaker as an antagonist, since it produces only mild abstinence in addicts maintained on $30 \mathrm{mg}$ methadone per day. ${ }^{21}$ Even appropriate long-term use of opiates can gradually produce a low level of physical dependence, and this subclinical state can be unmasked by administration of an opioid antagonist. It seems prudent to avoid agonist-antagonists in patients with significant prior use of morphine, meperidine, oxycodone, etc.

In theory, administration of an opioid antagonist might create problems if one later switched to a pure agonist (e.g., nalbuphine premedication followed by fentanyl intraoperatively). In practice, this interaction has not been a problem.

\section{Chronic use/abuse}

At present pentazocine is the only agonist-antagonist available in an oral formulation (sublingual buprenorphine is marketed in the UK). It is possible to give these drugs parenterally for long periods of time, but there is little clinical data on such use. Tolerance and physical dependence can occur, but the withdrawal syndrome is usually brief and not like that of morphine.

The World Health Organization has reviewed the abuse of agonist-antagonists and concluded that there is no basis for instituting narcotic controls. There has been very little abuse of the kappa type agonists, and addicts tend to avoid them. Pentazocine tablets were abused parenterally for a short time along with the antihistamine tripellenamine (so-called "T's and Blues"). The problem was solved by a reformulation in 1983. The subject of agonistantagonist abuse has recently been reviewed. ${ }^{22}$

\section{Conclusions}

The agonist-antagonists are effective analgesics with some significant differences from the pure agonists. The clinical indications for these two classes overlap but are not identical. All of the agonist-antagonists have excellent safety profiles: life-threatening respiratory depression is uncommon, and minor toxic effects like nausea, constipation, and urinary retention occur less frequently than with morphine. Several of these agents have been widely available for over ten years, and during that time they have been subject to remarkably little diversion or abuse.

There are at least three promising applications for these drugs in anaesthesia:

1 The kappa type drugs appear to be useful as sedatives and adjuncts to local or regional anaesthesia. We need better dosage guidelines for this indication and more data on interactions with other sedative drugs like benzodiazepines.

2 Epidural butorphanol has already shown some promise in obstetrics. A single dose provides relief of post- 
Caesarean pain for six to ten hours without pruritus or excessive sedation.

3 Finally, these analgesics may have advantages for Patient Controlled Analgesia, since they do not require locked pumps or narcotic inventory control. If the use of agonist-antagonists can significantly reduce pharmacy paperwork, their total costs will probably compare favourably with morphine and meperidine.

\section{References}

1 Lasagna $L$, Beecher $H K$. The analgesic effectiveness of nalorphine and nalorphine-morphine combinations in man. J Pharmacol Exp Ther 1954; 112: 356-363.

2 Wikler A, Fraser $H F$, Isbell $H$. N-allylnormorphine: effects of single doses and precipitation of acute "abstinence syndromes" during addiction to morphine, methadone or heroin in man (postaddicts). J Pharmacol Exp Ther 1953; 109: 8-20.

3 Keats AS, Telford J. Studies of analgesic drugs. X. Respiratory effects of narcotic antagonists. J Pharmacol Exp Ther 1966; 151: 126-32.

4 Martin WR, Eades CG, Thompson JA, Huppler RE, Gilbert PE. The effects of morphine- and nalorphinelike drugs in the non-dependent and morphine-dependent chronic spinal dog. J Pharmacol Exp Ther 1976; 197: 517-32.

5 Houde $R W$, Wallenstein $S L$. Clinical studies of morphinenalorphine combination. Fed Proc 1956; 15: 440-1.

6 Abboud TK, Moore M, Zhu J, et al. Epidural butorphanol or morphine for the relief of post-cesarean section pain: ventilatory responses to carbon dioxide. Anesth Analg 1987; 66: 887-93.

7 Stanley $T H$, Reddy $P$, Gilmore S, Bennett $G$. The cardiovascular effects of high-dose butorphanol-nitrous oxide anaesthesia before and during operation. Can Anesth Soc J 1983; 30: 337-41.

8 Murphy MR, Hug CC. The enflurane sparing effect of morphine, butorphanol and nalbuphine. Anesthesiology 1982; 57: 489-92.

9 Houde RW. Analgesic effectiveness of the narcotic agonist-antagonists. Br J Clin Pharmacol 1979; 7: 297S-308S

10 Kaiko RF, Wallenstein SL, Rogers AG, Canel A, Jacobs B, Houde $R W$. Intramuscular meptazinol and morphine in postoperative pain. Clin Pharmacol Ther 1985; 37: 58996.

11 Bacon BR, Marshall JB. Randomized, prospective, double-blind clinical trial of butorphanol and diazepam in patients undergoing upper gastrointestinal endoscopy. Acute Care 1988; 12(Suppl 1): 57-62.

12 Nagashima H, Karamanian A, Malovany R, et al. Respir atory and circulatory effects of intravenous butorphanol and morphine. Clin Pharmacol Ther 1976; 19: 738-45.
13 Gal TJ, Di Fazio CA, Moscicki J. Analgesic and respiratory depressant activity of nalbuphine: a comparison with morhine. Anesthesiology 1982; 57: 367-74.

14 Heel RC, Brogden RN, Speight TM, Avery GS. Buprenorphine: a review of its pharmacological properties and therapeutic efficacy. Drugs 1979; 17: 81-110.

15 Malis JL, Rosenthale ME, Gluckman MI. Animal pharmacology of WY-16225, a new analgesic agent. J Pharmacol Exp Ther 1975; 194: 488-98.

16 Radnay PA, Duncalf D, Novakovic M, Lesser ML. Common bile duct pressure changes after fentanyl, morphine, meperidine, butorphanol, and naloxone. Anesth Analg 1984; 63: 4.

17 Alderman EL, Barry WH, Graham AF, Harrison $D C$. Hemodynamic effects of morphine and pentazocine differ in cardiac patients. New Eng J Med 1972; 27 : 623-7.

18 Popio KA, Jackson DH, Ross AM, Schreiner BF, Yu PN. Hemodynamic and respiratory effects of morphine and butorphanol. Clin Pharm Ther 1978; 23: 281-7.

19 Aldrete JA, de Campo T, Usubiaga LE, Renck R, Suzuki D, Witt WO. Comparison of butorphanol and morphine as analgesics for coronary bypass surgery: a double-blind, randomized study. Anesth Analg 1983; 62: 78-83.

20 Latasch $L$, Probst $S$, Dudziak R. Reversal by nalbuphine of respiralory depression caused by fentanyl. Anesth Analg 1984; 63: 814-6.

21 Preston KL. Bigelow GE, Liebson IA. Butorphanolprecipitated withdrawal in opioid-dependent human volunteers. J Pharm Exp Ther 1988; 246: 441-8.

22 Johanson $C E$, Yanagita $T$ (Eds.). WHO Symposium on Drug Dependence: Benefit-Risk Ratio Assessment of Agonist-Antagonist Analgesics. Drug Alcohol Dep. 1987; 20: 289-409. 